\title{
Stacked-Ring Electrostatic Ion Guide
}

\author{
Shenheng Guan and Alan G. Marshall \\ Center for Interdisciplinary Magnetic Resonance, National High Magnetic Field Laboratory and Department of \\ Chemistry, Florida State University, Tallahassee, Florida, USA
}

In 1969 Bahr, Gerlich, and Teloy introduced an rf device that consisted of a stack of ring electrodes, with charge sign alternation between neighboring rings, to store or transport ions. Here we propose to operate such a device with electrostatic potentials rather than $\mathrm{rf}$ potentials: ions that move axially along the center of the guide are thereby subjected to an oscillating electrical potential similar to the sinusoidal rf potential in familiar rf-only multipole ion guides. The oscillating potential of the stacked-ring static ion guide focuses ions by exerting a field gradient force on the ions so as to push ions toward the central axis where the field is weakest. The stacked-ring ion guide produces an effectively static "pseudopotential" that is much steeper at the edge (potential varies as $e^{r}$ ) compared to a quadrupole or octupole guide (for which the potential varies as $r^{2}$ or $r^{6}$, where $r$ is radial position) and that is much flatter near the center of the guide (for potentially higher ion flux). Advantages of the new ion guide include static rather than $\mathrm{rf}$ potential, low electrical noise, a large field-free region near the central axis of the guide, and simple mechanical construction. A disadvantage of the stacked-ring ion guide is that high ion axial kinetic energy is required; ions with axial kinetic energy that is too low may be trapped in the shallow pseudopotential well between adjacent ring electrodes. (J Am Soc Mass Spectrom 1996, 7, 101-106)

$\mathrm{E}$ lectrospray ionization (ESI) [1] has revolutionized the mass analysis of biological macromolecules. By promoting large biopolymers with as many as tens of thousands of elemental charges into the gas phase, ESI has the capability to produce intact proteins and DNA quasimolecular ions with mass-tocharge ratios typically of a few thousand units per elementary charge. At or below that mass-to-charge ratio, Fourier transform ion cyclotron resonance mass spectrometry (FT-ICR/MS or FTMS) [2-12] can provide ultrahigh mass resolving power, accurate mass measurement, high sensitivity by means of repeated remeasurement, multistage tandem mass spectrometry, and long ion storage period for study of ionmolecule reaction chemistry. The combination of FTICR/MS and ESI shows great promise for biomolecules: accurate mass by direct measurement, primary structure by tandem mass spectrometry, conformations by $\mathrm{H}-\mathrm{D}$ exchange, and so forth. The efficient interface of an ESI source with a Fourier transform ion cyclotron resonance (FT-ICR) instrument faces two technical difficulties. First, ions are created in an ESI source at atmospheric pressure, but they have to be detected at $\leq 10^{-8}$ torr in a FT-ICR ion trap. Several stages of pumping usually are needed to overcome more than 10 orders of magnitude difference in pressure [13-16].

Address reprint requests to Professor Alan G. Marshall, Center for Interdisciplinary Magnetic Resonance, National High Magnetic Field Laboratory, Florida State University, 1800 East Paul Dirac Drive, Tallahassee, FL 32310.
Second, due to narrow access to the bore of a typical FT-ICR superconducting magnet, it is generally more convenient to generate ions outside the magnet. However, an external ion source creates the problem of transporting ions of a broad range in velocity and mass-to-charge ratio through the so-called "magnetic mirror" as ions pass into the strong-field region of the magnet on their way to the ion cyclotron resonance (ICR) ion trap. Thus it is necessary to maintain narrow focusing of the ions so that (1) ions may pass through the small conductance limit orifice at each stage of differential pumping and (2) ions may avoid the "magnetic mirror" by keeping close to the central axis of the solenoidal magnet's field.

In contrast to a mass or energy analyzer, an ion transport system or an ion guide should not discriminate among ions of different mass-to-charge ratio and/or kinetic energy. Rather, an ideal ion guide should generate an actual or effective potential minimum on the axis along which ions travel; that is, a two-dimensional potential well to confine ions radially but that allows ions to move freely along the axial direction. Unfortunately, Laplace's equation disallows the generation of such a potential minimum in free space by applying static voltages to the electrodes of a finite-length ion guide. However, ions may be transported through a hollow cylinder with a more negatively biased wire running down its central axis (Figure 1, top) [17-21]. Although the wire ion guide has the advantage of simple construction, ions without sufficient initial transverse velocity may be neutralized 


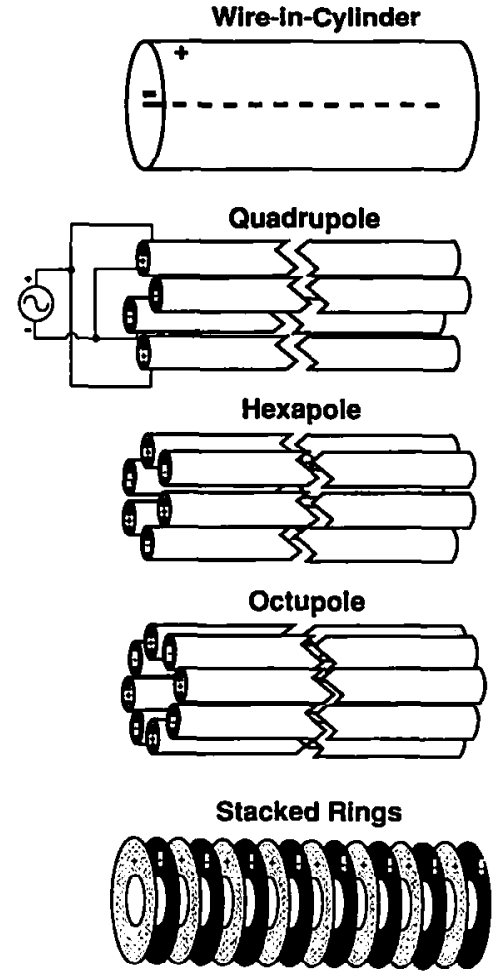

Figure 1. Various ion guide designs. In the stacked-ring guide, ions traveling at constant speed along the central axis of the stack are subjected to periodic potential oscillations, which have an effect qualitatively similar to the time-varying potential obtained by applying $\mathrm{rf}$ voltages between adjacent rods of a multipole ion guide (see text).

by striking the central wire. Moreover, the confining or focusing potential has an undesirable radial profile (see subsequent text).

The shortcomings of an electrostatic ion guide may be overcome by use of an ion guide based on a spatially inhomogeneous rf potential [22]. Ion motion in an inhomogeneous rf electric field may be analyzed into a fast oscillation of the ion at the frequency of the rf field and a much slower ion drift motion toward weaker-field regions. The slow motion is called ion secular motion and may be treated as if the ion moved in an equivalent electrostatic potential, designated as the "effective" or "pseudopotential" [23]. The general mathematical analysis and historical development of the pseudopotential formalism may be found in an excellent review by Gerlich [22]. Commonly used rf ion guides include rf-only multipoles, such as quadrupole, hexapole, or octupole ion guides (see Figure 1) $[24,25]$. In a multipole ion guide, the pseudopotential in the plane perpendicular to the axis is generated by $\mathrm{rf}$ voltages applied differentially between each pair of neighboring electrodes. An important feature of a multipole ion guide is that both the instantaneous and pseudopotentials are truly two dimensional (i.e., the potential is invariant to translation along the guide axis).
Closely related to the rf-multipole ion guide is the rf-only ion guide introduced by Bahr et al. [26], which consists of a stack of ring electrodes. When an rf voltage is applied between each pair of neighboring electrodes, an ion at rest on-axis is subjected to an oscillating electric field whose time-varying instantaneous amplitude varies with axial and radial location as shown in Figure 2. The time-averaged effect of a rapidly time-varying $\mathrm{rf}$ potential on a slow-moving ion is well represented by a static "pseudopotential" that is nearly flat near the central axis of the stack and that increases steeply with increasing radial displacement away from the axis (see Figure 3). Because of its nearly flat, steep-walled pseudopotential well, the rf-only ring ion guide has a broad field-free region near the central axis and is an excellent tool to study low temperature ion-molecule reactions.

Conventionally, the effective or pseudopotential concept is considered to arise from the effect of a rapidly oscillating $\mathrm{rf}$ potential field on an ion that moves slowly or is at rest. In this article we propose an "inverse" approach, in which rapidly moving ions pass through a stack of rings to which alternately positive and negative static voltages are applied. In the inertial frame of the moving ion, the potential at the ion will vary approximately sinusoidally with time
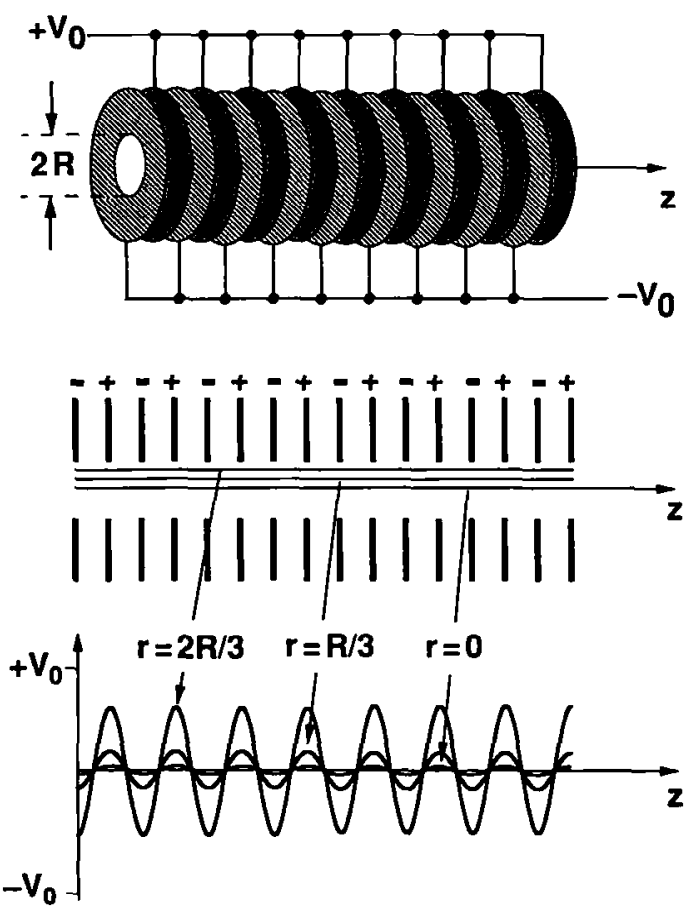

Figure 2. Schematic view of the voltages applied to the elements of a stacked-ring ion guide (top) and the potential as a function of axial position (bottom) that correspond to each of three radial offsets (middle). Note the approximately sinusoidal variation in potential as a function of axial position (bottom diagram), which results in an approximately sinusoidal variation of potential with time in the inertial frame of an ion traveling at constant speed along each of the three paths shown in the middle diagram. 


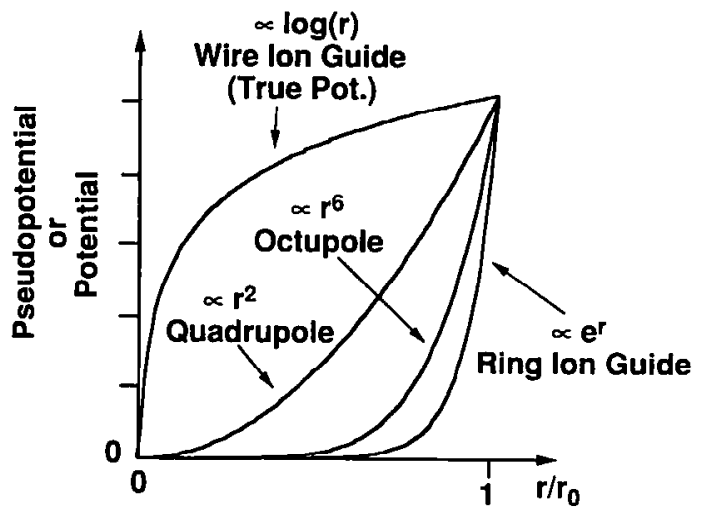

Figure 3. Comparison of radial electrostatic potential of a wire ion guide and the pseudopotentials of multipole and stacked-ring ion guides versus ion radial position ( $r_{n}$ is the distance from the central axis of the guide to the nearest outer electrode surface). Note that the stacked-ring guide produces the broadest and flattest electric field-free region, which provides a near "particlein-a-box" potential to confine ions as they travel through the guide.

as the ion passes through successive rings. Ions are thus subjected to a field gradient force that points toward the central axis where the electric "pseudofield" is weakest. The analysis of ion motion inside the stacked rings is therefore the same as that for an $\mathrm{rf}$ guide except that the alternating spatially inhomogeneous electric field is generated by an ion that moves past static-potential electrodes of successively opposite sign rather than a time-oscillating potential applied to ions that move slowly or are at rest.

Our present approach is conceptually similar to Courant, Livingston, and Snyder's magnetic-focusing synchroton, in which a series of magnetic quadrupoles is used to confine a high energy proton beam. The major advantages of the new stacked-ring ion guide are its simple mechanical construction and the employment of dc electrostatic potentials. Use of $\mathrm{dc}$ rather than high amplitude rf voltage is particularly attractive for an external ion source FT-ICR/MS instrument, in which ion detection is vulnerable to electrical noise in the same frequency range. We report the transport capabilities of the stacked-ring guide, based on simulated ion trajectories.

\section{Electrostatic Potential and Pseudopotential of a Ring Ion Guide}

\section{Electrostatic Potential of a Ring Electrode Ion Guide}

The following analysis of potentials of an rf ring ion guide is based on the work of Gerlich [22]. The potential boundary condition for an ideal cylindrical ring ion guide may be written in cylindrical coordinates as

$$
\Phi\left(r_{0}, z\right)=\Phi_{0} \cos \left(z / z_{0}\right)
$$

in which $r_{0}$ is the radius of the ring electrode and $z_{0}$ is the length of each ring element. For an rf ion guide, $\Phi_{0}$ varies sinusoidally with time, but for the present problem $\Phi_{0}$ may be treated as a constant. The potential inside the ion guide may be found by standard variable separation techniques [27]. Introduction of the reduced variables $\hat{z}=z / z_{0}$ and $\hat{r}=r / z_{0}$ enables us to express the potential as

$$
\Phi(r, z)=\Phi_{0}\left[I_{0}(\hat{r}) / I_{0}\left(\dot{\vec{r}}_{0}\right)\right] \cos (\hat{z})
$$

in which $I_{0}(x)$ is the zero-order modified Bessel function [28]. The corresponding electric field can be obtained as

$$
\begin{aligned}
\mathrm{E}(r, z)= & -\nabla \Phi(r, z) \\
= & \frac{\Phi_{0}}{z_{0}}\left(-\left[\frac{I_{1}(\hat{r})}{I_{0}\left(\hat{r}_{0}\right)}\right] \cos (\hat{z}) \mathbf{n}_{\mathbf{r}}\right. \\
& \left.+\left[\frac{I_{0}(\hat{r})}{I_{0}\left(\hat{r}_{0}\right)}\right] \sin (\hat{z}) \mathbf{n}_{z}\right)
\end{aligned}
$$

in which $\mathbf{n}_{\mathrm{r}}$ and $\mathbf{n}_{z}$ are unit vectors along the radial and axial directions. A special property of the modified Bessel function [28], $\left(d I_{0}(x)\right) / d x=I_{1}(x)$, has been used to derive the electric field. Because it is always more experimentally convenient to construct an ion guide by use of a series of electrodes with constant potential surfaces, the potential eq 2 may be realized by forming electrodes whose surfaces follow the curves

$$
I_{0}\left(r / z_{0}\right) \cos \left(z / z_{0}\right)=\text { constant }
$$

The isopotential contours that correspond to eq 4 are shown in Figure 4 (left).

\section{Pseudopotential of a Ring Ion Guide}

The general concept of a pseudopotential or effective potential has been discussed in detail by Gerlich [22]. Briefly, in an inhomogeneous ac field, ion motion may be treated as the sum of a slow drift motion and a fast oscillating motion. The drift motion is due to the inhomogeneity of the ac field and may be considered as if the ion is moving in an electrostatic potential or pseudopotential. If the ac instantaneous field can be written as

$$
\mathrm{E}(r, t)=\mathbf{E}_{0}(r) \cos \Omega t
$$

in which $\Omega$ is the angular frequency of the rf signal, then the pseudopotential takes the form

$$
V^{*}(\mathbf{r})=q^{2}\left|\mathbf{E}_{0}(\mathbf{r})\right|^{2} / 4 m \Omega^{2}
$$

in which $q$ and $m$ are the charge and mass of the ion. Clearly, the pseudopotential $V^{*}(\mathbf{r})$ depends on the mass of the ion and is independent of the polarity of 
Instanteneous Potential
Pseudopotential

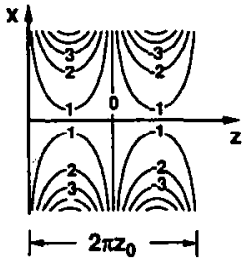

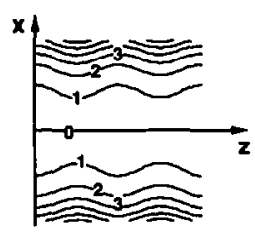

Adlabatioity Parameter

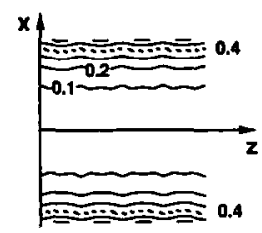

Figure 4. Properties of an electrostatic ring ion guide that extend for one repeat unit (two oppositely charged adjacent rings) along the stack. Left: Electrostatic potential produced by rings whose shape is specified by eq 4. Middle: Pseudopotential in the inertial frame of an ion traveling down the stacked-ring axis. Right: "Adiabaticity" parameter $\eta$. Empirically, the pseudopotential approximation is valid, provided that the adiabaticity parameter is less than 0.4 . Thus, ions whose trajectories do not extend beyond the iso- $\eta=0.4$ contours may be described by ion motion (in the $z$ direction) in a slow-varying pseudopotential (middle diagram) rather than in the fast-varying actual potential (left diagram).

the charge. For an rf ring ion guide, $\Phi_{0}$ is given by

$$
\Phi_{0}=V_{0} \cos \Omega t
$$

in which $V_{0}$ is the rf amplitude and the potential difference between neighboring electrodes is $2 V_{0}$ $\cos \Omega t$. Substituting eq 3 into eq 6 , we obtain the pseudopotential of an $\mathrm{rf}$ ring electrode ion guide,

$$
V^{*}(\mathbf{r})=\frac{q^{2} V_{0}^{2}}{4 m \Omega^{2} z_{0}^{2}}\left(\frac{I_{1}^{2}(\hat{r}) \cos ^{2}(\hat{z})+I_{0}^{2}(\hat{r}) \sin ^{2}(\hat{z})}{I_{0}\left(\hat{r}_{0}\right)}\right)
$$

which is shown in Figure 4 (middle).

\section{Stable Operation of an rf Ion Guide-Adiabaticity}

For a simple rf device such as a two-dimensional if quadrupole ion guide, the conditions required for stable operation of the ion guide (i.e., ions with stable orbits that remain within the device) may be derived from a "stability" diagram. Furthermore, one may use the pseudopotential approximation with the q-factor $q_{x}=q_{y}=-4 q V / m r_{0}^{2} \Omega^{2}<0.4$, in which $r_{0}$ is the distance from the axis to a rod surface, $V$ is the rf voltage amplitude, and $\Omega$ is the rf frequency. For a more general case such as a ring ion guide, it is not possible to produce a "stability" diagram without extensive ion trajectory simulations. Fortunately, the conditions under which the pseudopotential approximation is valid depend on a so-called adiabaticity criterion. The basic idea is to find conditions under which the average energy of an ion in an rf field is an "adiabatic" constant of the motion; namely, that the slow ion motion may be separated from the fast motion. For an adiabaticity parameter $\eta$, defined as

$$
\eta=2 q|\nabla| \mathrm{E}_{0}(\mathbf{r})|| / m \Omega^{2}
$$

Dehmelt [29] proved that for a quadrupole potential field, $\eta$ reduces to a constant $q$ factor. For any other device, the parameter $\eta$ is no longer constant as the ion position changes. Nevertheless, one can still evaluate $\eta$ along the trajectory of a given ion. If the condition

$$
\eta<0.4
$$

is met, the pseudopotential approximation remains valid [22].

\section{Pseudopotential and Adiabaticity for an Ion that Moves in a Stationary Electric Field}

The foregoing discussion of pseudopotential and adiabaticity is based on the assumption that the ion is at rest and the electrical field oscillates with time. Conversely, suppose that we now consider a moving ion in an electrostatic field. Let the ion move at constant axial speed $v_{z}$ along the central axis of the ion guide so that the ion axial kinetic energy is $m v_{z}^{2} / 2$. If the axial kinetic energy of the ion is significantly higher than the deviation in local electrical potential anywhere along the ion trajectory, then the time-average ion axial kinetic energy will be constant because the ion encounters an effectively time-periodic potential surface. Thus, the spatial $z$ periodicity of the static potential electrode stack produces a time-periodic potential in the inertial frame of an ion traveling at constant speed along the $z$ axis. With this correspondence, we may apply the preceding analysis to determine the transport behavior of the ion guide.

The question now becomes how to interpret the if frequency. The ion travels at $v$, meters per second along the $z$ axis. According to eq 1 , the spatial periodicity of the potential in the $z$ direction is $2 \pi z_{0}$. Therefore, the ion will pass $N=2 v_{z} / 2 \pi z_{0}$ electrodes per second, which corresponds to $N / 2$ periodic changes per second in potential to which the ion is subjected. The equivalent frequency of potential changes can be written as

$$
\nu_{\mathrm{eff}}=v_{z} / \pi z_{0}
$$

in hertz or

$$
\Omega_{\text {eff }}=2 \pi \nu_{\text {eff }}=2 v_{z} / z_{0}
$$

in radians per second. The pseudopotential for a fastmoving ion traveling through an electrostatic ring ion guide may then be obtained by substituting eq 12 into eq 8 to yield

$$
V^{*}(\mathbf{r})=\frac{q^{2} V_{0}^{2}}{32 E_{z}}\left(\frac{I_{1}^{2}(\hat{r}) \cos ^{2}(\hat{z})+I_{0}^{2}(\hat{r}) \sin ^{2}(\hat{z})}{I_{0}\left(\hat{r}_{0}\right)}\right)
$$

in which $E_{z}=m v_{z}^{2} / 2$ is the ion axial kinetic energy. The "adiabaticity" factor $\eta$ may be estimated from eq 
9; $\Omega$ taken from eq 12. Although the $\eta$ factor depends on ion position, $\eta$ may be calculated (as shown in Figure 4, right). If an ion trajectory is limited to a spatial region where $\eta<0.4$, the pseudopotential approximation will be valid. Because the variation of pseudopotential along the $z$ axis is small and varies steeply with radius near the ring electrodes, an ion behaves essentially like a hard sphere traveling down a cylindrical pipe.

\section{Simulated Ion Trajectories}

Ion trajectory simulations were carried out with Simion version 6.0 , for a stack of 84 conductive ring electrodes, each with a 6-mm-diameter flat-surface hole and 2-mm space between rings. Although such a ring surface contour does not match the optimum shape given by eq 4 , the potential nevertheless still approximates the desired limiting form near the symmetry axis of the guide. In all simulations, a static potential of $\pm 50 \mathrm{~V}$ was applied to each pair of neighboring ring electrodes.

For example, a singly charged ion of $1000 \mathrm{u}$ and axial kinetic energy of $35 \mathrm{eV}$ travels at $2.6 \times 10^{3} \mathrm{~m} / \mathrm{s}$. As the ion passes through the stacked rings, the potential in the inertial frame of the ion corresponds to an effective frequency $\Omega_{\text {eff }}$ of $1.3 \times 10^{6} \mathrm{rad} \mathrm{s}$ or $\nu_{\mathrm{eff}}=207$ $\mathrm{kHz}$. The effective rf frequency decreases dramatically with increasing ion mass, because a heavier ion of a given kinetic energy has smaller velocity. For example, the frequency drops to $6.7 \mathrm{kHz}$ for a singly charged ion of the same axial kinetic energy ( $35 \mathrm{eV})$ but with mass of $10^{6} \mathrm{u}$. Ions of sufficiently low axial kinetic energy may be trapped near the negatively biased ring electrodes.

Figure 5 shows the effect of axial kinetic energy on ion trajectories through the stacked rings. High mass ions must be excited to high axial kinetic energy for efficient transport through the stack. For example (Figure 5, top), singly charged ions of $10^{6} u$ that enter the stack with incident polar angle of $\leq 10^{\circ}$ (with respect to the central axis of the stacked rings) are transmitted successfully if the axial kinetic energy is $\geq 50 \mathrm{eV}$. However, the axial kinetic energy requirement is substantially lower for singly charged ions of $10^{3} \mathrm{u}$ (Figure 5, bottom).

The ion guide has an injection acceptance area of at least $0.04 A$ (i.e., $0.2^{2}$ ), in which $A$ is the area of ion guide cross-section area. For example, ions of mass $10^{6}$ $\mathrm{u}$ and total kinetic energy of $35 \mathrm{eV}$ may enter the ring stack either significantly off-axis or with incident polar angle up to at least $15^{\circ}$ (see Figure 6).

\section{Conclusion}

Based on the present theory and simulations, the electrostatic ring ion guide appears to offer excellent ion transmission characteristics. The greatest advantage of
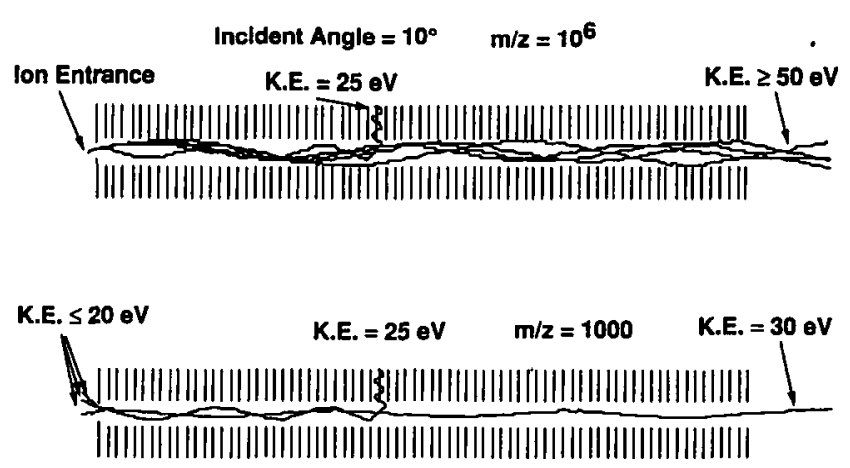

Figure 5. Effect of ion axial kinetic energy on ion trajectories through a stacked-ring ion guide (see text). Note that ions of $m / z$ $10^{6}$ (or $\mathrm{m} / z 10^{3}$ ) pass successfully through the guide for ion axial energy $\geq 50 \mathrm{eV}$ (or $30 \mathrm{eV}$ ).

the device is that it uses only electrostatic potential and therefore eliminates arcing and noise from the if signal. Moreover, the steep pseudopotential well also should result in less $\mathrm{rf}$ heating than multipole-type $\mathrm{rf}$ ion guides. Furthermore, the instantaneous potential is truly cylindrically symmetric, so that there is no unwanted cyclotron excitation in an external ion source FT-ICR instrument as can occur for multipole-based ion guides. Finally, the mechanical construction of the ion guide is simple.

An important difference between the stacked-ring guide and a multipole ion guide is that the rf frequency applied to a multipole ion guide is obviously independent of ion mass-to-charge ratio, whereas the effective $r f$ frequency for a stacked-ring guide depends on ion velocity (and thus, in general, on ion mass). However, the present results show that isoenergetic singly charged ions of widely different mass (1000$1,000,000 \mathrm{u}$ ) are nevertheless successfully transmitted through the guide if they are accelerated initially to sufficiently high axial kinetic energy. It is important to accelerate ions to a high axial velocity to avoid trapping ions in the shallow pseudopotential well between adjacent ring electrodes. Finally, in the special situation that ions of different mass have nearly the same velocity (as for matrix-assisted laser desorption/ionization [30]), the stacked-ring guide should perform
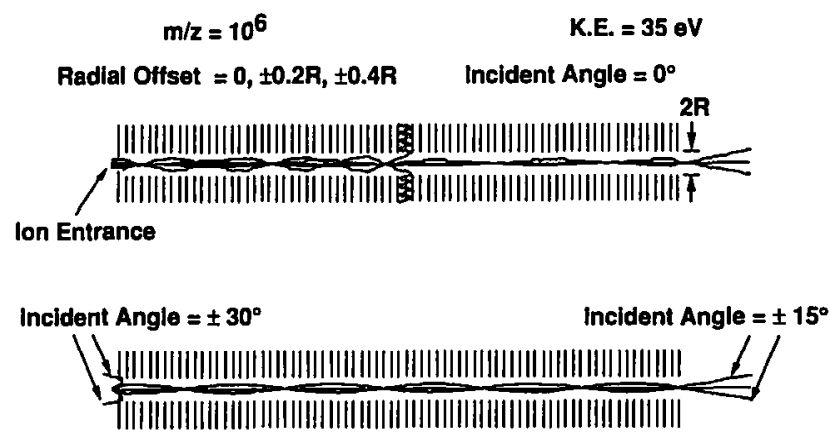

Figure 6. Effect of ion injection offset (top) and polar angle (bottom) with respect to the stacked-ring central axis on ion trajectory through a stacked-ring electrostatic ion guide (see text). 
comparably well for ions of widely different mass-tocharge ratios.

\section{Acknowledgments}

This work was supported by the National Science Foundation (CHE-94-13008) and the National High Magnetic Field laboratory at Florida State University. We thank David Dahl for providing us with an advance copy of Simion 6.0 for ion trajectory simulations.

\section{References}

1. Whitehouse, C. M.; Dreyer, R. N.; Yamashita, M.; Fenn, J. B. Anal. Chem. 1985, 57, 675-679.

2. Marshall, A. G.; Schweikhard, L. Int. I. Mass Spectrom. Ion Processes 1992, $118 / 119,37-70$.

3. Köster, C.; Kahr, M. S.; Castoro, J. A.; Wilkins, C. L. Mass Spectrom. Rev. 1992, 11, 495-512.

4. Speir, J. P.; Gorman, G. S.; Amster, I. J. In Mass Spectrometry in the Biological Sciences: A Tutorial; M. L. Gross, Ed.; Kluwer Academic Publishers: Dordrecht, The Netherlands, 1992; pp 199-212.

5. Jacoby, C. B.; Holliman, C. L.; Gross, M. L. In Mass Spectrometry in the Biological Sciences: A Tutorial; M. L. Gross, Ed.; Kluwer Academic Publishers: Dordrecht, 1992; pp 93-116.

6. Nibbering, N. M. M. Analyst 1992, 117, 289-293.

7. Buchanan, M. V.; Hettich, R. L. Anal. Cltem. 1993, 65, 245A-259A.

8. Brenna, J. T.; Creasy, W. R.; Zimmerman, J. Amer. Chem. Soc. Symp. Ser. 1993, 236, 129-154.

9. Guan, S.; Kim, H. S.; Marshall, A. G.; Wahl, M. C.; Wood, T. D.; Xiang, X. Chem. Rev. 1994, 8, 2161-2182.

10. Mclafferty, F. W. Acc. Chem. Res. 1994, 27, 379-386.

11. Wilkins, C. L., Ed. Trends Anal. Chem. 1994, 13, 223-251 (Special Issue: Fourier Transform Mass Spectrometry).

12. Holliman, C. L.; Rempel, D. L.; Gross, M. L. Mass Spectrom. Rev. 1994, 13, 105-132.
13. Henry, K. D.; Williams, E. R.; Wang, B.-H.; McLafferty, F. W.; Shabanowitz, J.; Hunt, D. F. Proc. Natl. Acad. Sci. U.S. A. 1989, 86, 9075-9078.

14. Hofstadler, S. A.; Schmidt, E.; Guan, Z.; Laude, D. A., Jr. J. Am. Soc. Mass Spectrom. 1993, 4, 168-176.

15. Ijames, C. F.; Markey, S. P. J. Am. Soc. Mass Spectrom. 1994, $5,398-406$.

16. Winger, B. E.; Hofstadler, S. A.; Bruce, J. E.; Udseth, H. R.; Smith, R. D. J. Amer. Soc. Mass Spectrom. 1993, 4, 566-577.

17. Kingdon, K. H. Plyys. Rev. 1923, 21, 408-418.

18. Wolf, B.; Mudgett, P. D.; Macfarlane, R. D. I. Amer. Soc. Mass Spectron. 1990, 1, 28-36.

19. Solouki, R.; Gillig, K. J.; Russell, D. H. Anal. Chem. 1994, 66, 1583-1587.

20. Limbach, P. A.; Marshall, A. G.; Wang, M. Int. J. Mass Spectrom. Ion Processes 1993, 125, 135-143.

21. Marto, J. A.; Guan, S.; Limbach, P. A.; Wahl, M. C.; Marshall, A. G. In Proceedings of the 41st ASMS Conference on Mass Spectrometry and Allied Topics; San Francisco, CA, 1993; pp $471 \mathrm{a}, \mathrm{b}$.

22. Gerlich, D. In State-Selected and State-to-State Ion-Molecule Reaction Dynamics. Part 1. Experiment, Vol. LXXXII; C. Y. Ng and M. Baer, Eds.; Wiley: New York, 1992; pp 1-176.

23. Major, F. G.; Dehmelt, H. G. Phys. Rev. 1968, 170, 91-107.

24. Wolinik, H. Optics of Charged Particles; Academic Press: San Diego, CA, 1987; p 293.

25. March, R. E.; Hughes, R. J. Quadrupole Storage Mass Spectrometry; Wiley: New York, 1989; p 471.

26. Bahr, R.; Gerlich, D.; Teloy, E. Verhandl. DPG (VI) 1969, 4, 343.

27. Jackson, J. D. Clnssical Electrodynamics; Wiley: New York, 1975; $\mathrm{p} 848$.

28. Abramowitz, M.; Stegun, I. A., Eds. Handbook of Mathematical Functions with Formulas, Graphs and Mathematical Tables, Vol. 55, 10th ed.; US Dept. of Commerce: Washington, DC, 1972.

29. Dehmelt, H. G. Adv. At. Mol. Phys. 1967, 3, 53-72.

30. Beavis, R. C.; Chait, B. T. Chem. Phys. Lett. 1991, 181, 479-484. 